\title{
Computational Modelling of Cardiac Metabolism in Atrial Myocytes
}

\author{
Funebi Francis Ijebu ${ }^{1,4}$, Qince $\mathrm{Li}^{1}{ }^{*}$, Kuanquan Wang ${ }^{1}$, Haibo Sui ${ }^{1}$, Lufang Zhou ${ }^{5}$, Yongfeng Yuan ${ }^{1}$, \\ Henggui Zhang ${ }^{1,2,3}$
}

\author{
${ }^{1}$ School of Computer Science and Technology, Harbin Institute of Technology, Harbin, China \\ ${ }^{2}$ School of Physics and Astronomy, University of Manchester, Manchester, UK \\ ${ }^{3}$ Space Institute of Southern China (Shenzhen), Shenzhen, China \\ ${ }^{4}$ Department of Computer Science, University of Uyo, Akwa Ibom State, Nigeria \\ ${ }^{5}$ School of Medicine, University of Alabama at Birmingham, Birmingham, AL, US
}

\begin{abstract}
In this study, a computational model elucidating effects of cytosolic metabolic processes on cardiac metabolism and excitation-contraction coupling of an atrial cell is developed. Kinetic and thermodynamic rate laws were applied to describe Michaelis-Menten dynamics of metabolites in glycolysis and tricarboxylic acid cycle. Our computational model links cytosolic metabolism to mitochondrial metabolism by passive diffusion and carrier mediated transport using law of mass action, in adherence to the selective permeability of the mitochondrial membrane to metabolites. Simulation results for metabolic substrates in both compartments under control conditions showed stable dynamics for excitation-contraction coupling of the heart. Further simulation of dynamic modulated cardiac workload was consistent with experimental and theoretical expectations of substrate variation under different conditions. The ability of our model to simulate cardiac energy demand-supply balance under varied conditions confirms its strength which can help to further explore mechanisms of the pathology of disease resulted from metabolic dysfunction and discover the possibility of therapeutic intervention.
\end{abstract}

\section{Introduction}

Computational models of cardiac metabolism developed in recent years for mammalian species have either focused on the ventricular cell [1] or only on the electrophysiology of the atrial cells [2]. The atrial cell differs slightly from the ventricular cell in terms of structure and nature of its ionic currents [3]. In addition, the T-tubules in ventricular cells penetrate and transmit action potentials and calcium $\left(\mathrm{Ca}^{2+}\right)$ deep into the interior for excitation-contraction coupling [4]. But its absence in atrial cell leads to inadequate propagation of
$\mathrm{Ca}^{2+}$ released from the sarcoplasmic reticulum, hence leading to failures of excitation-contraction (EC) coupling in central regions of the atrial cell. Such differences in structural and ionic build-up have been reported to cause changes in membrane currents [5], this suggests that bioenergetics in the atrial cell might have different roles on EC coupling.

Adenosine triphosphate (ATP) is the currency for cardiac EC coupling and it is synthesized in the mitochondria by a series of $\mathrm{Ca}^{2+}$ sensitive dehydrogenase reactions [6]. The substrate, acetyl-CoA that initiates these reactions is provided as an end product mainly from glycolysis. Whereas the dehydrogenases exist freely and catalyze mostly unidirectional reactions to produce energy in the mitochondria, glycolytic enzymes in the cytosol exist in supramolecular complexes and catalyze mostly bidirectional reactions leading to energy consumption [7]. Several computational models have been constructed to study and explain these processes. However; current models linking glycolysis to mitochondrial dehydrogenases $[6,8]$ do not explicitly account for all the enzyme reactions in the pathways. Therefore, atrial metabolism and its effect on cardiac bioenergetics have not been properly characterized. In this study, we present a computational model (Figure 1) elucidating the biochemical processes that lead to stable cardiac metabolism. By focusing on aerobic substrate oxidation and ATP synthesis in the mitochondria, we study the energy demand-supply balance to investigate the effects of glycolytic reactions in the atrial cell on cardiac excitation-contraction coupling.

\section{Methods}

Our model was compartmented into the cytosolic and mitochondrial domains, with single energy (ATP) pool. Descriptions of metabolic processes of our model use michaelis-menten equations. The metabolic rates were defined by twelve ordinary differential equations (ODEs) solved with the $\mathrm{C}$ programmed integrator, CVODE. 


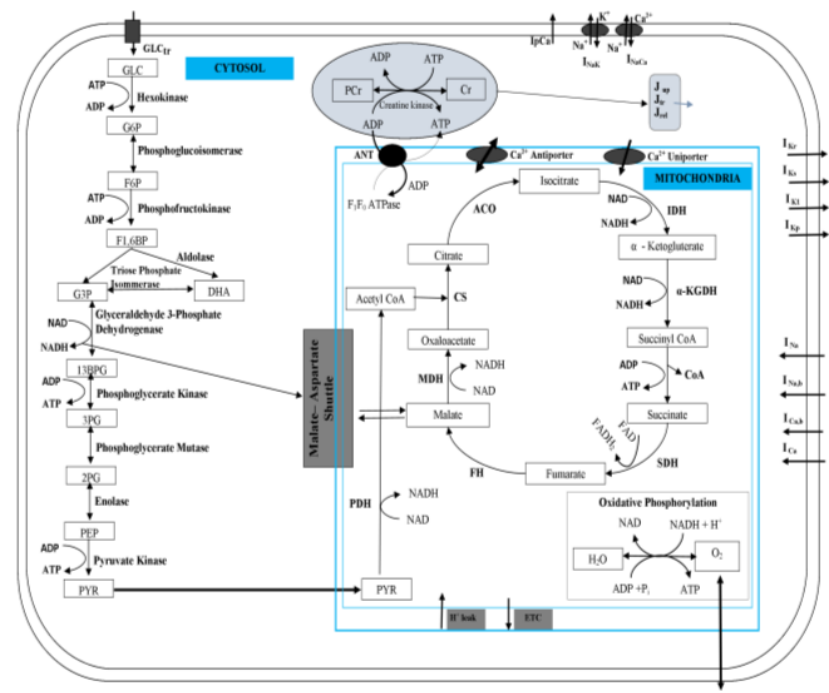

Figure 1: Schematics of our computational model of cardiac metabolism. Glucose enters the cytosol through the glucose transporter. This inflowing glucose quickly undergoes phosphorylation and gets converted over the series of steps terminating at pyruvate. This end product is then transported by the pyruvate transporter into the mitochondria, where pyruvate dehydrogenas e (PDH) oxidizes it to acetyl-CoA which combines with oxaloacetate to initiate dehydrogenase reactions resulting in cellular ATP production.

Due to reversibility in some glycolytic reactions, the enzyme rates in these reactions were defined as in equation 1, fulfilling laws of mass action and thermodynamic equilibrium. Reactions catalyzed by phosphoglucoisomerase, triose phosphate isomerase, glyceraldehyde 3-phosphate dehydrogenase, phosphoglycerate kinase, phosphoglycerate mutase and enolase were modeled in this form.

$$
V_{E}=\frac{V_{m f} \frac{\left[S_{1}\right]}{K_{m}^{S 1}} \cdot \frac{\left[S_{2}\right]}{K_{m}^{S 2}}-V_{m r} \frac{\left[P_{1}\right]}{K_{m}^{p 1} \cdot \frac{\left[P_{2}\right]}{K_{m}^{P 2}}}}{1+\frac{\left[S_{1}\right]}{K_{m}^{S 1}}+\frac{\left[S_{2}\right]}{K_{m}^{S 2}}+\frac{\left[S_{1}\right]}{K_{m}^{S 1}} \cdot \frac{\left[S_{2}\right]}{K_{m}^{S 2}}+\frac{\left[P_{1}\right]}{K_{m}^{P 1}}+\frac{\left[P_{2}\right]}{K_{m}^{P 2}}+\frac{\left[P_{1}\right]}{K_{m}^{P 1}} \cdot \frac{\left[P_{2}\right]}{K_{m}^{P 2}}}
$$

where, $V_{E}$ is the rate of enzyme, $\left[S_{1}\right],\left[S_{2}\right]$ and $\left[P_{1}\right],\left[P_{2}\right]$ are substrates and products concentrations respectively. $V_{m f}$ and $V_{m r}$ are maximum forward and reverse rates of the reaction respectively, while $K_{m}$ is the Michaelis constant of the corresponding element.

Equation 2 was used to define irreversible reactions such as reactions catalyzed by hexokinase, phosphofructokinase, aldolase, and pyruvate kinase.

$$
V_{E}=\frac{V_{m f} \cdot \frac{\left[S_{1}\right]}{K_{m}^{S 1}} \cdot \frac{\left[S_{2}\right]}{K_{m}^{S 2}}}{1+\frac{\left[S_{1}\right]}{K_{m}^{S 1}}+\frac{\left[S_{2}\right]}{K_{m}^{S 2}}+\frac{\left[S_{1}\right]}{K_{m}^{S 1}} \cdot \frac{\left[S_{2}\right]}{K_{m}^{S 2}}}
$$

When competitive product inhibition was reported for a reversible or irreversible reaction, the component $\left(1+\frac{[P]}{K_{i}^{P}}\right)$ was incorporated into the denominator of the equation; where $[P]$ is the concentration of the product and $K_{i}^{P}$ is the inhibition constant. Glucose uptake into the cytosol was modeled as in Bakker model [9] while pyruvate transporter was modified from Teusink model [10]. For both transporters, passive diffusion was assumed. However, to model the membrane impermeable transport of cytosolic $\mathrm{NADH}$ to the mitochondria, the carrier mediated malateaspartate shuttle in previous study [11] was modified and integrated.

\section{Results}

All integrated substrates in both cytosol and mitochondria attained steady state within the first fifty seconds of simulation and maintained this peak throughout the simulation time (Figure 1). The stability in dynamics of metabolites and ATP satisfies the demand-supply balance of energy in the cell under physiological conditions. Under this state, the energy produced from cardiac metabolism is used for mechanical work, ionic homeostasis, signaling, and synthesis of various cellular molecules. Therefore, any unwarranted alteration to this metabolic stability leads to changes in the electrical and mechanical activities of the heart which may cause various cardiac disease conditions.

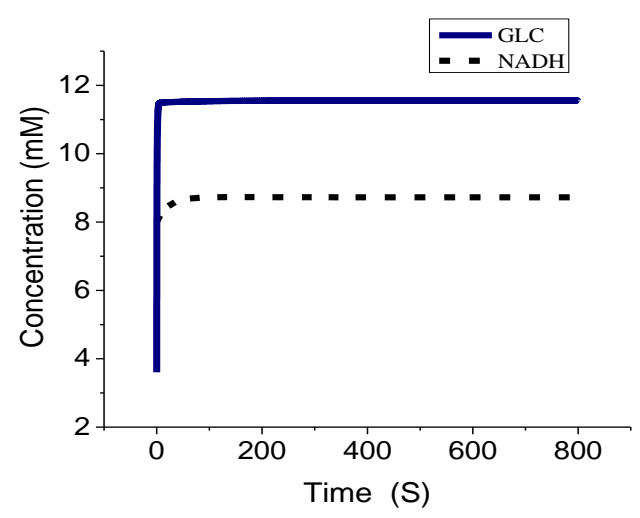

Figure 2: Steady state simulation results for glycolytic substrates and mitochondrial NADH.

Theoretically, cardiac work rate should be minimal and fairly stable during rest, as is also predicted by our present model, however, in cases of shock and sudden fright; cardiac energy demands are abrupt and requires immediate response. Our model simulated both conditions and the results for the glycolytic and TCA cycle substrates responses is as presented in figure 4 and figure 5 respectively. Rest and 
exercise or fright conditions were simulated by dynamic switch of cardiac pacing frequency as described in Figure 4, the $0.25 \mathrm{~Hz}$ corresponds to normal pacing of the heart, while $2.0 \mathrm{~Hz}$ represents exercise or fright condition which results in increased cardiac workload and energy demand. The increase in glucose transporter rate is reflected by the increase in glucose concentration while all other glycolytic substrates concentration decreased (Figure 4). This glucose dynamics agrees with experimentally observed results in guinea pig heart [6], and in spontaneously hypertensive rat myocytes [12].

When the work load increases, energy production decreases as shown in Figure 4A and cardiac efficiency reduces. The decrease in the efficiency of utilizing energy for mechanical work affects the potential of the heart to perform ionic homeostasis because the mechanical work of the heart and its ionic homeostasis are strongly linked by a complex set of electrical and biochemical mechanisms [13, 14]. Therefore, a sustained pacing of the heart at a high frequency over a longer period might result in a cardiac collapse descriptive of cardiovascular disease conditions like heart failure and atrial fibrillation. When the work load increases, a large overshoot of mitochondrial NADH in our model may be a result of the parallel activation of the malate-aspartate shuttle integrated in our model but absent in previous models [15]. The activation of the NADH shuttle increases the proton influx from the cytosol, hence, the reduced undershoot and relatively larger overshoot.
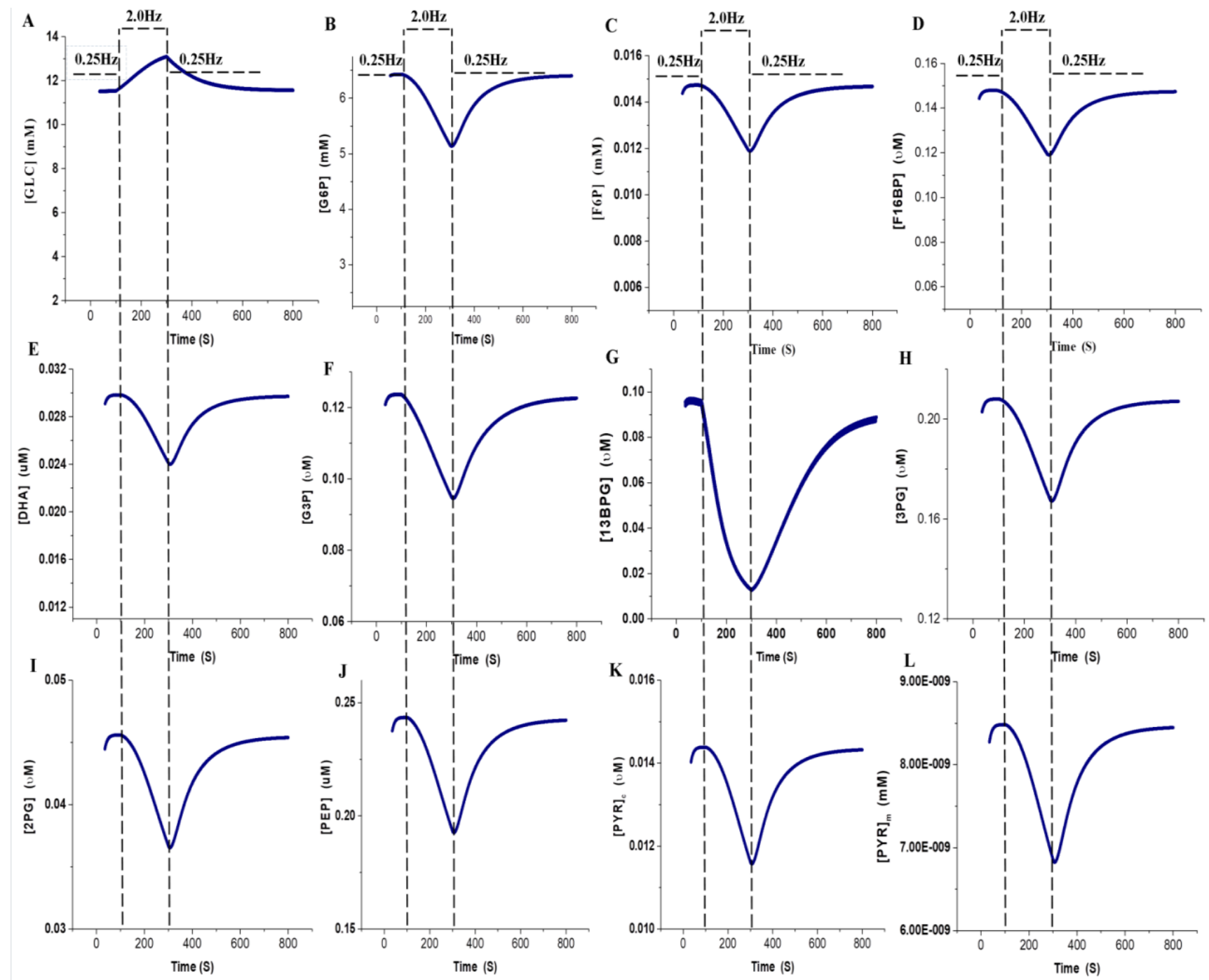

Figure 3: Glycolytic substrates and mitochondrial pyruvate response to modulated cardiac workload, with $0.25 \mathrm{~Hz}(0-100 \mathrm{~S}$, and $300-800 \mathrm{~S})$ as baseline frequency and $2.0 \mathrm{~Hz}(100-300 \mathrm{~S})$ as high frequency. 

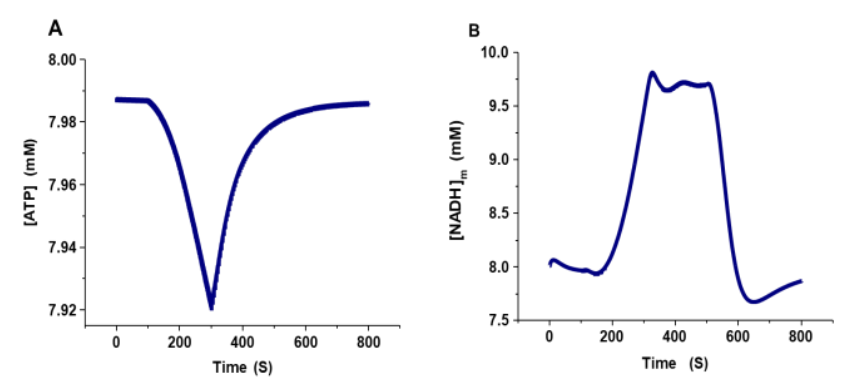

Figure 4: Modulated cardiac workload response for (A) ATP (B) mitochondrial NADH.

\section{Conclusion}

In this study, we presented an integrated kinetic model of cardiac metabolism in the atrial cell and simulated the impact on cardiac bioenergetics. With a normal cardiac pacing frequency of $0.25 \mathrm{~Hz}$, our model reproduced steady state dynamics for all cytosolic and mitochondrial metabolic substrates. Further contributions of this study include the quantitative evidence of parallel activation of cytosolic and mitochondrial metabolic processes during abrupt dynamic switch of cardiac workload. Model simulation of experimentally observed increase in intracellular glucose concentration and decrease in ATP concentration upon increase in cardiac energy demand

\section{Acknowledgements}

The work is supported by the National Nature Science Foundation of China (NSFC) (No. 61601143, 81770328, 61572152, and 61571165), Heilongjiang and China Postdoctoral Science Foundation under Grant nos.2015M581448 (to QL), the Natural Science Foundation of Shandong Province (No. ZR2017MF051), the Science Technology and Innovation Commission of Shenzhen Municipality (No. JSGG20160229125049615 and JCYJ20151029173639477).

\section{References}

[1] Cortassa S, Aon A.M, Marba E, Winslow L.R and O'Rourke B. An integrated model of cardiac mitochondrial energy metabolism and calcium dynamics,. Biophysical Journal 2003, 84:2734-2755.

[2] Favaron $M$ and Bernardi P. Tissue-specific modulation of the mitochondrial calcium uniporter by magnesium ions. FEBS LETTERS. 1985.82(2): 260 - 264.

[3] Grandi E, Pandit V.S, Voigt N, Workman J.W, Dobrev D, Jalife J, and Bers M.D. Human atrial action potential and $\mathrm{ca}^{2+}$ model sinus rhythm and chronic atrial fibrillation. Circ Res. 2011, 109:1055-1066.
[4] Bootman D.M, Higazi R.D, Coombes S, and Roderick L.H. Calcium signaling during excitation-contraction coupling in mammalian atrial myocytes. Journal of Cell Science 2006, 119: 3915-3925.

[5] Hüser J, GaoWang Y, Sheehan A.K, Cifuentes F, Lipsius L.S and Blatter A.L. Functional coupling between glycolysis and excitationcontraction coupling underlies alternans in cat heart cells. Journal of Physiology 2000, 524(3):795 - 806.

[6] Zhou L, Cabrera E.M, Okere C.I, Sharma N, and Stanley C.W. Regulation of myocardial substrate metabolism during increased energy expenditure: insights from computational studies. Am. J Physiol. Heart Circ. Physiol. 2006, 291: H1036-H1046.

[7] Ventura-Clapier R, Garnier A and Veksler V. Energy metabolism in heart failure. J Physiol. 2003, 555(1):1-13.

[8] Lambeth J.M and Kushmerick J.M. A computational model for glycogenolysis in skeletal muscle. Annals of Biomedical Engineering 2002, 30:808-827.

[9] Bakker M.B, Michaels M.A.P, Opperdoes R.F and Westerhoff V.H. Glycolysis in bloodstream form Trypanosoma Brucei can be understood in terms of the kinetics of the glycolytic enzymes. The Journal of Biological Chemistry 1997, 272:3207-3215.

[10] Teusink B, Passarge J, Reijenga A.C, Esgalhado E, van der Weijden C.C, Schepper M, Walsh C.M, Bakker M.B, van Dam K, Westerhoff V.H and Snoep L.J. Can yeast glycolysis be understood in terms of in vitro kinetics of the constituent enzymes? Testing Biochemistry. Eur. J. Biochem 2000, 267:5313-5329.

[11] Lu M, Zhou L, Stanley C.W, Cabrera E.M, Saidel M.G, and Yu X. Role of the malate-aspartate shuttle on the metabolic response to myocardial Ischemia. Journal of Theoretical Biology 2008, 254:466475.

[12] Zhou L, Salem E.J, Saidel M.G, Stanley C.W and Cabrera E.M. Mechanistic model of cardiac energy metabolism predicts localization of glycolysis to cytosolic subdomain during ischemia. Am J Physiol. - Heart Circ. Physiol., 2005. 288: H2400-H241

[13] Nickel A, LÖffler J, and Maack C. Myocardial energetics in heart failure. Basic Res. Cardiol. 2013. 108(358):1 - 20.

[14] Masoud G.T.W, Clanachan S.A, and Lopaschuk D.G. The failing heart: is it an inefficient engine or an engine out of fuel? In: Cardiac Remodeling - Molecular Mechanisms, Advances in Biochemistry in Health and Disease 5 by Jugdutt B.I and Dhalla N.S (eds.). Springer. 2013: $65-84$

[15] Cortassa S, Aon A. M, O’Rourke B, Jacques R, Tseng H-J, Marba E and Winslow L. R. A computational model integrating electrophysiology, contraction, and mitochondrial bioenergetics in the ventricular myocyte. Biophysical Journal. 2006, 91:1564-1589.

Address for correspondence.

Qince Li

Xinjishu Building 901,

Harbin Institute of Technology

Xidazhi Street, Nangang District,

Harbin,

China

qinceli@hit.edu.cn 